\title{
The general set in the MCIM Isotopic Model
}

\author{
R. M. Falcón and J. Núñez
}

\begin{abstract}
To obtain a bigger number of mathematical and physical applications of the Santilli's isotheory, the latest studies have shown the necessity of analyzing isotopic models which use non associative laws. The main goal of this paper is to give a generalization of the isotopic construction model based on the multiplication (MCIM), which is useful to obtain non associative mathematical isostructures.
\end{abstract}

Keywords: Santilli's Isotheory, isotopic model, isostructure.

Mathematical subject classification: 03H05, 08A05, 03C65.

\section{Introduction}

In 1978, R. M. Santilli proposed a generalization of the conventional Lie's theory by using isotopies. The isotopies of Lie's theory were constructed to lift the theory from its current sole applicability to linear systems to nonlinear systems, lifting achieved via the reconstruction of linearity on isospaces over isofields. It was the first stage of what is actually known as Santilli's Isotheory [1]. He considered that the basic unit $I$ of each mathematical structure can depend on several factors external to the system in which we are placed, like coordinates, speed, time, density, temperature, and so on. It involves an isounit of the type $\widehat{I}=\widehat{I}(x, v, t, \mu, \tau, \ldots)$. By using this principle, Santilli carried out a step by step construction which generalizes the most common mathematical structures, originating those denominated mathematical isostructures [2], [3]. It allowed him to progress in the development of some physical applications, mainly in Quantum Mechanics and Dynamics of Particles [4].

In 2001, the isotopic construction model based on the multiplication (from now on, it will be denoted by MCIM) was introduced by ourselves (see [5]) in the same way as the one proposed by Santilli, although by putting a special emphasis in the use of $*$-laws. Later, it was improved in [6] and [7]. Nevertheless, to enlarge the number of mathematical isostructures and to get new practical applications, it is necessary to weaken the associativity hypothesis, obtaining in this way more

Received 7 July 2004. 
general cases. To do it, all the elements which form the general set of every isotopy must be settled. This will be then the main objective of this paper.

\section{Preliminary Concepts}

From now on an isotopic lifting or isotopy will be any correspondence between a mathematical structure and another one of the same type, that is to say, in such a way that both verify the same properties. Note that according to this definition an isotopy could not be a map. The image is then called isotopic structure or isostructure [1].

Santilli's isotopic model of 1978 is based on the generalization of the initial unit: $I \rightarrow \widehat{I}=\widehat{I}(x, v, t, \mu, \tau, \ldots)$. So, fixed any mathematical structure $E$, endowed with an inner law $\times$, this model considers a set $V \supseteq E$, endowed with an associative law $*$ and $I, \widehat{I}, T \in V$, where $I \in E$ is the unit of $*$ in $V$ and $T=\widehat{I}^{-I} . V, T$ and $\widehat{I}$ are respectively called general set, isotopic element and isounity of the isotopy. So, it is defined the isostructure $\widehat{E}$, endowed with the law $\widehat{\widehat{x}}$ with unit $\widehat{I}$ as:

$$
\begin{gathered}
\widehat{\widehat{E}}=\{\widehat{\widehat{x}}=x * \widehat{I}: x \in E\}, \\
\bar{a} \overline{\times} \bar{b}=\bar{a} * T * \widehat{\vec{b}}=(a * b) * \widehat{I}, \text { for all } \widehat{\widehat{a}}, \bar{b} \in \bar{E} .
\end{gathered}
$$

The MCIM isotopic model generalizes the Santilli's one, by using as many *-laws as the initial ones in $E$, in such a way that if $E$ is endowed with an inner law $\circ$, it will have associated a $*$-law $\star$, such that:

$$
\overline{\widehat{a} \widehat{\circ} \widehat{b}}=(a \star b) * \widehat{I}, \text { for all } \bar{a}, \bar{b} \in \widehat{\widehat{E}} .
$$

Let us observe that if $\star \equiv *$, then $\widehat{\widehat{o}} \equiv \overline{\widehat{x}}$ is the Santilli's previous law. So, any isotopy is given in the following way:

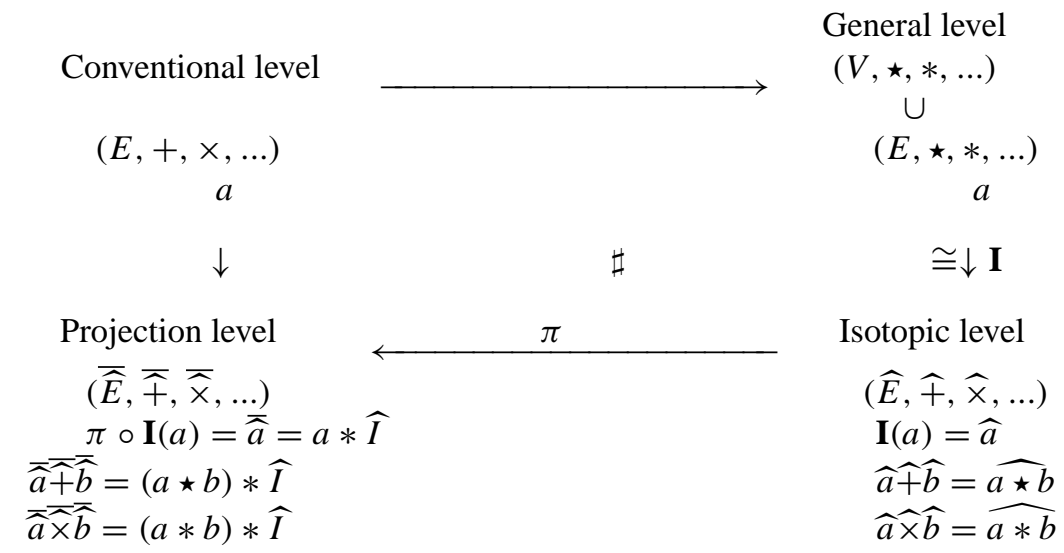




\section{A Generalization of the MCIM}

\subsection{A first generalization of the MCIM}

To get a generalization of the MCIM, we are going to weaken some of the conditions which are necessary when using this isotopic model. To do it, let us consider the frame of hypothesis:

(1) A set $E$, endowed with an inner law o, a set $V \supseteq E$, endowed with a inner law $*$, with unit $I \in V$, and an arbitrary element $\widehat{I}$ in $V$.

So, let us define:

$$
\widehat{\widehat{E}}=\{\widehat{\widehat{x}}=x * \widehat{I}\}, \quad \overline{\widehat{a} \widehat{\circ} \bar{b}}=(a * b) * \widehat{I} .
$$

We will say that $\bar{E}$ is injective if $\bar{a}=\bar{b} \in \overline{\widehat{E}}$ implies $a=b \in E$.

Let us observe that if $*$ is associative and there exists $T=\widehat{I}^{-I} \in V$, then we have the MCIM isotopic model. In this case, $\widehat{\widehat{E}}$ will be injective.

In the general case, we have the following result:

Proposition 2.1. If $*$ is associative in $V$, then $\widehat{\widehat{o}}$ is associative in $\widehat{V}$, provided it is well-defined, that is, if for all $a, b, c \in E$ such that $\bar{a}=\widehat{b}$, it is verified that $\overline{\widehat{a} \widehat{\circ} \widehat{c}}=\widehat{\widehat{b} \widehat{o c}}$ and $\overline{\widehat{c} \widehat{o} \widehat{a}}=\overline{\widehat{c} \widehat{o}} \widehat{b}$. Moreover, if $\widehat{E}$ is injective, the converse is also verified.

Proof. Let $*$ be associative. As $\widehat{\widehat{O}}$ is well-defined, it means that such a law has a perfect sense over $\widehat{E}$. So $\widehat{o}$ is associative:

$$
(\overline{\widehat{a} \widehat{\circ} \widehat{b}}) \overline{\widehat{o c}}=((a * b) * c) * \widehat{I}=(a *(b * c)) * \widehat{I}=\overline{\widehat{a} \widehat{\circ}}(\overline{\bar{b}} \overline{\widehat{o c}}) .
$$

Let us now suppose that $\overline{\widehat{o}}$ is associative and $\widehat{\widehat{E}}$ is injective. So:

$$
((a * b) * c) * \widehat{I}=(\overline{\widehat{a} \widehat{\circ} \widehat{b}}) \overline{\widehat{o c}}=\overline{\widehat{a} \widehat{\circ}}(\overline{\widehat{b}} \widehat{\widehat{\circ} \hat{c}})=(a *(b * c)) * \widehat{I} .
$$

Now, as $\widehat{E}$ is injective, we have that $(a * b) * c=a *(b * c)$ and thus, $*$ is injective.

Let us observe that if $\widehat{\widehat{E}}$ is injective, then $\overline{\mathrm{o}}$ is well-defined. However, as we will see next, the converse is not true in general. Indeed, let us suppose that $\widehat{E}$ is not injective. So, they should exist, at least, two different elements $x, y \in E$ 
such that $x * \widehat{I}=y * \widehat{I}$. Besides, as $\widehat{o}$ should be well-defined, it must be $(x * a) * \widehat{I}=(y * a) * \widehat{I}$, for all $a \in E$. If we also consider that $E$ has the inverse element property with respect to $*$, we can take $a=x^{-I} \in E$, and so:

$$
\widehat{I}=\left(y * x^{-I}\right) * \widehat{I} .
$$

Then, let us observe that if $*$ is associative in $V$, it will imply the non existence of $T=\widehat{I}^{-I}$, because in the other case, by multiplying on the right by $T$ in the last equality, it is deduced that $I=y * x^{-I}$; this is, $x=y$, which is a contradiction.

On the other hand, if $*$ is non associative in $V$, the corresponding isotopic element could exist or not, although if it exists, then the following equality has to be satisfied:

$$
I=\left[\left(y * x^{-I}\right) * \widehat{I}\right] * T .
$$

or equivalently:

$$
T=\left[\left(y * x^{-I}\right) * \widehat{I}\right]^{-I} .
$$

So, we have then proved the following result:

Proposition 2.2. By adding in (1) that $E$ has the inverse element property with respect to $*$ and that $\widehat{E}$ is not injective, then either $T=\widehat{I}^{-I}$ does not exist or if it exists, then $*$ is non associative in $V$. Besides, in this case, for all $x, y \in E$ such that $\bar{x}=\widehat{\hat{y}}$, it is verified that $T=\left[\left(y * x^{-I}\right) * \widehat{I}\right]^{-I}$.

\subsection{A second generalization of the MCIM}

Let us now consider the following set of hypothesis:

(2) A set $E$, endowed with two inner laws $\circ$ and $\bullet$, a set $V \supseteq E$, endowed with two inner laws, $*$, with unit $I \in V$, and $\star$, and arbitrary elements $\widehat{I}, T$ in $V$.

Let us define:

$$
\begin{aligned}
\overline{\widehat{E}} & =\{\overline{\widehat{x}}=x * \widehat{I}\}, \\
\widehat{\widehat{a} \widehat{\circ} \widehat{b}} & =[(\overline{\widehat{a}} * T) *(\widehat{b} * T)] * \widehat{I}, \\
\overline{\widehat{a} \widehat{\bullet}} & =[(\widehat{\widehat{a}} * T) \star(\widehat{b} * T)] * \widehat{I} .
\end{aligned}
$$

Let us observe that if $*$ is associative and there exists $T=\widehat{I}^{-I} \in V$, then we have the MCIM isotopic model. 
This generalization is useful to study some of the properties that the general set $V$ should verify. To see it, we will show first an explicit example. We are going to get the complex numbers structure $(\mathbb{C},+, \cdot)$ as an isotopic projection of the real numbers structure $(\mathbb{R},+, \times)$. To do it, we will employ the laws $\star_{\mid \mathbb{R}} \equiv+$ and $*_{\left.\right|_{\mathbb{R}}} \equiv \times$ and the isounit $\widehat{I}=\widehat{I}(t)$, depending on a factor "time" $t \in \mathbb{R}$, such that $x * \widehat{I}(t)=x+t i \in \mathbb{C}$, for all $x, t \in \mathbb{R}$. In this way, $\overline{\mathbb{R}}=\mathbb{C}$.

The following step is to get + and $\cdot$ as laws in the projection level. To achieve this objective, it will be necessary to use again the time factor, although now in an inverse sense with respect to the procedure used to obtain the isotopic set in question. For this reason, a good way to deal with this aspect would be to use the isotopic element $T=T(t)=\widehat{I}(t)^{-1}$. So:

$$
\begin{aligned}
& (a+b i) \overline{\widehat{+}}(c+d i)=([(a+b i) * T] \star[(c+d i) * T]) * \widehat{I}, \\
& (a+b i) \overline{\hat{*}}(c+d i)=([(a+b i) * T] *[(c+d i) * T]) * \widehat{I} .
\end{aligned}
$$

Then, we can take advantage that the isotopic element depends on the time to conserve the following useful information: fixed $(a+b i) \in \mathbb{C}$, this element was just obtained when the time is $t=b$. To get it, we can define the operation $*$ in the general set $V$ as follows:

$$
(a+b i) * T=a_{b}
$$

where $a_{b}$ (which could be identified with the pair $(a, b)$ in $\mathbb{R}^{2}$ ) would belong to $V$. So, it is useful to consider the following set:

$$
\mathbb{C}_{T}=\left\{a_{b}=(a+b i) * T: a, b \in \mathbb{R}\right\} .
$$

Remark. In a similar way, if we are now considering an isotopy of any structure $E$, it will be useful to consider the set:

$$
E_{T}=\{\bar{a} * T: \bar{a} \in \bar{E}\} .
$$

Therefore, the corresponding general set $V$ can be defined as:

$$
V=E \cup \widehat{E} \cup E_{T} \cup\{\widehat{I}, T\} .
$$

Now, at this point, it is important to note that in the case of the MCIM isotopic model, sets $E$ and $E_{T}$ coincide. It is so because under this model the associativity of the operation $*$ in the general set $V$ and the existence of the isotopic element $T=\widehat{I}^{-I}$ are satisfied by hypothesis. So, for all $a \in E$ it is verified:

$$
(a * \widehat{I}) * T=a *(\widehat{I} * T)=a .
$$


In particular, this proves the necessity of generalizing such a model to the following three cases: first, when the associativity of $*$ in $V$ is not verified. Second, when the isotopic element does not exist and third, when none of these two conditions is satisfied. These three cases will be dealt in this paper.

On the other hand, it would be necessary to define the corresponding $*$-laws between elements of $E_{T}$. By example, in the concrete case which we are dealing, it would be convenient to define the laws $\star$ and $*$ in $\mathbb{R}_{T}=\left\{a_{b}: a, b \in \mathbb{R}\right\}$ as follows:

$$
a_{b} \star c_{d}=(a+c)_{b+d}, \quad a_{b} * c_{d}=((a \times c)-(b \times d))_{b \times c+a \times d}
$$

It would be also necessary to define the operation $*$ between elements of $\mathbb{R}_{T}$ and the isounit $\widehat{I}$ :

$$
a_{b} * \widehat{I}=a+b i
$$

In this way, we would obtain in the projection level:

$$
\begin{gathered}
(a+b i) \overline{+}(c+d i)=([(a+b i) * T] \star[(c+d i) * T]) * \widehat{I}= \\
=\left(a_{b} \star c_{d}\right) * \widehat{I}=\left((a+c)_{b+d}\right) * \widehat{I}=(a+c)+(b+d) i . \\
(a+b i) \overline{\widehat{\times}}(c+d i)=([(a+b i) * T] *[(c+d i) * T]) * \widehat{I}= \\
=\left(a_{b} * c_{d}\right) * \widehat{I}=\left(((a \times c)-(b \times d))_{b \times c+a \times d}\right) * \widehat{I}= \\
=(a c-b d)+(b c+a d) i .
\end{gathered}
$$

Note that, in particular, we have obtained that:

$$
(\widehat{\widehat{\mathbb{R}}}, \overline{\boldsymbol{+}}, \overline{\widehat{x}})=(\mathbb{C},+, \cdot)
$$

So, it is proved that an isofield, which conventionally cannot be doted of a total order, can be obtained starting from a totally ordered field, like real numbers, by using an isotopic lifting. In this way, it is also proved the necessity of considering the isoorder which was defined in [6]. Moreover, the study of the isoorder should be deeper in this concrete case, to solve some problems which appear. We will deal with this study in future works.

Finally, we can summarize the information relative to our general set as follows:

$$
V=\mathbb{R} \cup \mathbb{C} \cup \mathbb{R}_{T} \cup\{\widehat{I}, T\}
$$

being, for all $a, b, c, d \in \mathbb{R}$ :

$$
a \star b=a+b, \quad a * b=a \times b, \quad a * \widehat{I}(t)=a+t i
$$




$$
\begin{gathered}
(a+b i) * T=a_{b}, \quad a_{b} * \widehat{I}=a+b i, \quad a_{b} \star c_{d}=(a+c)_{b+d} \\
a_{b} * c_{d}=(a c-b d)_{a d+b c}, \quad \widehat{I} * T=T * \widehat{I}=I=1 \equiv 1_{0}
\end{gathered}
$$

An aspect to remark is that by using this construction we have shown an example in which the operation $*$ is non associative in $V$, since fixed $a \in \mathbb{R}$ and a certain instant of time $t_{0}$, one has:

$$
(a * \widehat{I}) * T=\left(a+t_{0} i\right) * T=a_{t_{0}} \neq a=a * I=a *(\widehat{I} * T)
$$

Besides:

$$
(a * T) * \widehat{I}=a_{0} * \widehat{I}=a=a *(T * \widehat{I})
$$

Moreover, if we demand the construction to be coherent, another condition to be satisfied would be the following:

$$
[(a * \widehat{I}) * T] * \widehat{I}=a * \widehat{I}
$$

As $T=\widehat{I}^{-I}$, it must be $\widehat{I}=I * \widehat{I}=(\widehat{I} * T) * \widehat{I}$ and thus, we get the Moufang's Identity:

$$
[(a * \widehat{I}) * T] * \widehat{I}=a *((\widehat{I} * T) * \widehat{I})
$$

\subsection{The MCGIM isotopic model}

We can try to generalize the MCIM isotopic model by considering that $T$ is not the inverse of the isounit $\widehat{I}$ in $V$. In this case, we will call generalized isotopic element to $T$, which is a different concept from isotopic element:

Definition 2.3. It will be said that an isotopy follows the generalized MCIM (from now on, MCGIM) when, under usual notations, there exists an element $T \in V$ such that, for all $a \in E$, it is verified:

$$
(\overline{\widehat{a}} * T) * \widehat{I}=\overline{\widehat{a}}=a * \widehat{I}
$$

So, the set $E_{T}$ acquires a great importance when we must decide the isotopy to construct, because such a set will be the one that has to verify properties satisfied by the isostructure to get. In this way, it is convenient to center us in the nature of the set $E_{T}$.

Proposition 2.4. Let us consider an isotopic lifting by using MCGIM. Then, $E=E_{T}$ if and only if for all $a \in E$, it is verified:

$$
(a * \widehat{I}) * T=a .
$$


Proof. The sufficient condition is evident. So, let us suppose $a \in E$. As $E=E_{T}$, there exists $b \in E$ such that $a=(b * \widehat{I}) * T$ and so, $a * \widehat{I}=$ $((b * \widehat{I}) * T) * \widehat{I}=b * \widehat{I}$. Thus, $a=(a * \widehat{I}) * T$.

This result allows us to have confidence in the generalization achieved, because all the basic aspects of isotopies are preserved. In fact, we are imposing the associativity of the operation $*$ at no time, which is particularly favorable for the study about the isotopical relationship between associative and non associative mathematical structures.

In the other way, note that in the particular case in which $*$ is associative in $V$ and $T=\widehat{I}^{-I}$ is the isotopic element, we got then the MCIM isotopic model as a particular case of the MCGIM one. Moreover, the following result is verified:

Proposition 2.5. By using the MCGIM isotopic model, if $*$ is an associative law in $V$, with unit $I$, then they are verified:

a) If there exists $a \in E$ such that $a * \widehat{I}$ has left inverse in $V$ with respect to $*$, then $T * \widehat{I}=I$.

b) If $E_{T}=E$ and there exists $a \in E$ admitting left inverse in $V$ with respect to $*$, then $\widehat{I} * T=I$.

In particular, if (a) and (b) are satisfied, then $T$ is the isotopic element of such an isotopy.

Proof. Note that the final assert of the proposition is evident, because the condition $T=\widehat{I}^{-I}$ is directly deduced from (a) and (b). So, it is sufficient to prove both items. Without lost of generality, we can suppose in both of them that $*$ is associative:

a) Let us suppose that there exists a left inverse of $a * \widehat{I}$ in $V$, for a given $a \in E$. Then:

$$
a * \widehat{I}=(\widehat{a} * T) * \widehat{I}=(a * \widehat{I}) *(T * \widehat{I}) \Rightarrow I=T * \widehat{I} .
$$

b) Let us now suppose that $E_{T}=E$ and that there exists a left inverse of $a \in E$ in $V$, with respect to $*$. Then:

$$
a=(a * \widehat{I}) * T=a *(\widehat{I} * T) \Rightarrow I=\widehat{I} * T .
$$


So, when working with associative $*$-laws, it is not more interesting the injectivity of the used isotopy but if $E=E_{T}$ or not. Nevertheless, what is the matter with isotopies in which $E \neq E_{T}$ ? They are possible, because we have already considered the case in which $\widehat{\mathbb{R}}=\mathbb{C}$ was obtained, being $\mathbb{R}_{T} \neq \mathbb{R}$. Such an example is useful to show that in every isotopy both properties and laws demanded for $\widehat{E}$ have to be satisfied in $E_{T}$, because such properties will be inherited by the isostructure to obtain. So, given an initial mathematical structure and another of the same type, every isotopic lifting consists on finding a superstructure $V$ which contains both structures plus an isounit and an generalized isotopic element. In this way, let us consider finally the following:

Example 2.6. Let us suppose the nilpotent group $\left(\mathbb{Z} / \mathbb{Z}_{2},+\right)$, with the usual sum. We are going to carry out an isotopic lifting of it by starting from elements of isotopy $\widehat{I}=0$ (associated with the isotopic element $T$ ) and $*$ defined both in such a way that, fixed $a, b \in \mathbb{Z} / \mathbb{Z}_{2}$, they are verified:

$$
a * b=a+b ; \quad a * \widehat{I}=\bar{a}=a+0=a
$$

In this way, we get $\overline{\widehat{\mathbb{Z}}_{\mathbb{Z}_{2}}}=\mathbb{Z} / \mathbb{Z}_{2}$. We also define, for all $a \in \mathbb{Z} / \mathbb{Z}_{2}$ :

$$
\begin{gathered}
\widehat{a} * T=a_{T}, \quad a_{T} * \widehat{I}=a, \quad 0_{T} * 0_{T}=0_{T}, \\
1_{T} * 1_{T}=1_{T}=1_{T} * 0_{T}=0_{T} * 1_{T} .
\end{gathered}
$$

So, fixed $a, b \in \mathbb{Z} / \mathbb{Z}_{2}$, we have in the projection level:

$$
\overline{\widehat{a}} \overline{+} \bar{b}=\pi \circ \mathbf{I}((\widehat{\vec{a}} * T) *(\widehat{b} * T))=\pi \circ \mathbf{I}\left(a_{T} * b_{T}\right) .
$$

Hence:

$$
0 \overline{\widehat{+}} 0=0, \quad 1 \overline{\widehat{+}} 1=1=1 \overline{\widehat{+}} 0=0 \overline{\widehat{+}} 1,
$$

and thus, $\left(\overline{\widehat{\mathbb{Z} / \mathbb{Z}_{2}}}, \overline{\widehat{+}}\right)=\left(\mathbb{Z} / \mathbb{Z}_{2}, \overline{\widehat{+}}\right)$ is a non nilpotent group.

So, in this way, a non nilpotent mathematical isostructure has been obtained as an isotopic projection of a nilpotent structure. However, it has been necessary for getting it to impose in our construction that the corresponding pair $\left(\left(\mathbb{Z} / \mathbb{Z}_{2}\right)_{T}, *\right)$ coincides with the isostructure searched. 


\title{
References
}

[1] R. M. Santilli, On a possible Lie-admissible covering of the Galilei Relativity in Newtonian Mechanics for nonconservative and Galilei noninvariant systems, Hadronic J. 1 (1978), 223-423. Addendum, ibid, 1 (1978), 1279-1342.

[2] R. M. Santilli, Isotopies of contemporary mathematical structures, I: Isotopies of fields, vector spaces, transformation theory, Lie algebras, analytic mechanics and space-time symmetries, Algebras, Groups and Geometries. 8 (1991), 169-266.

[3] G. T. Tsagas and D. S. Sourlas, Mathematical Foundations of the Lie-Santilli Theory, Hadronic Press (1993).

[4] R. M. Santilli, Elements of Hadronic Mechanics, Vol. I, Mathematical Foundations Second Edition, Kiev, 1995.

[5] R. M. Falcón Ganfornina and J. Núñez Valdés, La isoteoría de Santilli, Mathematical Series, International Academic Press, America - Europe - Asia, ISBN 1-57485-055-5 (2001).

[6] R. M. Falcón Ganfornina and J. Núñez Valdés, Studies of the Tsagas-SourlasSantilli isotopology, Algebras, Groups and Geometries. 20 issue 1 (2003), 1-100.

[7] R. M. Falcón Ganfornina and J. Núñez Valdés, Hausdorff isospaces and coordinatetemporal charts, Lagrange and Hamilton Geometries and their Applications [Radu Miron (Ed.)] 49 (2004), 115-123.

\author{
R. M. Falcón and J. Núñez \\ Departamento de Geometría y Topología \\ Facultad de Matemáticas \\ Universidad de Sevilla \\ Aptdo 1160, 41080 Sevilla \\ ESPAÑA
}

E-mail: rafalgan@us.es / jnvaldes@us.es 